\title{
Study of Parathyroid Hormone as an Independent Risk Marker of Heart Failure
}

\author{
${ }^{1}$ Siraj A Khan, ${ }^{2}$ Krishna M lyyapu, ${ }^{3}$ Kompella SS Sai Baba, ${ }^{4}$ Sreekanth Yerram
}

\begin{abstract}
Introduction: Heart failure (HF) is a clinical syndrome characterized by cardiac pump failure with signs and symptoms arising from salt and water retention. Heart failure is associated with considerable mortality and morbidity. Identification of modifiable risk factors may reduce incidence of HF and its complications. The aim of our study is to assess parathyroid hormone (PTH) as a risk marker for HF and its association with severity of HF.
\end{abstract}

Materials and methods: In this cross-sectional study, 120 subjects with HF were recruited and they were compared with 60 age- and sex-matched controls. Along with the routine parameters, N-terminal pro B-type natriuretic peptide (NT-proBNP), intact PTH, and vitamin D were estimated. The study group was divided into quartiles depending on $\mathrm{PTH}$ value.

Results: The median PTH (81.5 pg/mL) and NT-proBNP (3753 pg/ $\mathrm{mL})$ in HF patients are found to be significantly higher $(p<0.0001)$ when compared with control subjects. The median vitamin $D$ concentration $(18 \mathrm{ng} / \mathrm{mL})$ though low in cases is not statistically significant when compared with controls. Demographic, clinical, and laboratory characteristics are compared across the quartiles of PTH. Highest number of New York Heart Association (NYHA) class IV HF cases are found in highest quartiles of PTH. Logistic regression analysis demonstrated that high concentration of $\mathrm{PTH}$ [odds ratio of 1.1113 ; 95\% confidence interval (Cl) 1.07-1.14; $p<0.0001$ ] and low levels of vitamin D (odds ratio of 1.053; $95 \%$ Cl 1.0079-1.1009) are significantly associated with HF.

Conclusion: This study has demonstrated that higher concentration of PTH is associated with severe form of HF. Vitamin D deficiency is also seen in the study group.

Keywords: Heart failure, Parathyroid hormone, Vitamin D

How to cite this article: Khan SA, lyyapu KM, Sai Baba KSS, Yerram S. Study of Parathyroid Hormone as an Independent Risk Marker of Heart Failure. Indian J Med Biochem 2017;21(2):157-161.

\section{Source of support: Nil}

Conflict of interest: None

\footnotetext{
${ }^{1,2}$ Associate Professor, ${ }^{3}$ Professor, ${ }^{4}$ Assistant Professor

${ }^{1-3}$ Department of Biochemistry, Nizam's Institute of Medical Sciences, Hyderabad, Telangana, India

${ }^{4}$ Department of Cardiology, Nizam's Institute of Medical Sciences, Hyderabad, Telangana, India
}

Corresponding Author: Siraj A Khan, Associate Professor Department of Biochemistry, Nizam's Institute of Medical Sciences, Hyderabad, Telangana, India, Phone: +919908892371 e-mail: sakhan1994@yahoo.com

\section{INTRODUCTION}

Heart failure is a clinical syndrome characterized by cardiac pump failure with signs and symptoms arising from salt and water retention. Heart failure is also associated with systemic illness, especially in advanced stages characterized by oxidative stress in various tissues causing damage to soft tissues and bone. Heart failure is a major epidemic and a significant public health burden associated with considerable morbidity and mortality. ${ }^{1}$ It results not only from cardiac overload or injury but also from a complex relationship between genetic, neurohormonal, inflammatory, and biochemical changes. ${ }^{2}$

Hypertension and coronary artery disease are predominant causes of $\mathrm{HF}$ and account for $80 \%$ of all the cases. The well-recognized modifiable risk factors for HF are hypertension, impaired glucose tolerance, an elevated total and high-density lipoprotein cholesterol ratio, obesity, cigarette smoking, ${ }^{3}$ and other potential modifiable risk factors that include altered mineral metabolism, deficiency of vitamin D, stimulation of inflammatory cytokines, and oxidative stress. Identification of modifiable risk factor of HF may reduce incidence of $\mathrm{HF}$ and its complications.

Parathyroid hormone, a peptide hormone secreted by parathyroid gland, helps in calcium homeostasis. A decrease in concentration of calcium releases PTH, which enhances calcium reabsorption from ascending limb of loop of Henle and causes excretion of phosphorus in proximal duct. Secondly, it stimulates 1- $\alpha$-hydroxylation of 25-hydroxyvitamin D to 1, 25-dihyrdroxy vitamin D, which in turn causes reabsorption of calcium from the gut. Parathyroid hormone also acts on osteoclast causing bone resorption with release of phosphorus and calcium in the blood. ${ }^{4}$ In order to maintain serum calcium within a narrow range, PTH and vitamin D act together in response to changes in serum calcium levels. Decreasing vitamin D levels leads to increasing PTH concentrations. ${ }^{5}$

Many studies have shown that PTH has chronotropic ${ }^{6}$ and inotropic effects on cardiac muscle, along with a direct hypertrophic effect on cardiomyocytes. ${ }^{7}$

The aim of the present study is to assess PTH as a risk marker in $\mathrm{HF}$ and its association with severity of HF.

\section{MATERIALS AND METHODS}

In this cross-sectional study, 120 consecutive patients admitted in cardiac intensive care unit during the month 
of June and July 2016 in Nizam's Institute of Medical Sciences, Hyderabad, India, with the diagnosis of HF based on clinical features and echocardiography were recruited. For every patient age, sex, NYHA class, left ventricular ejection fraction (LVEF), and medication, along with the history of diabetes, hypertension, heart rate and rhythm, and cause of HF were noted. Subjects with vitamin D supplementation were excluded. In control group, 60 age- and sex-matched healthy controls were included. Random blood sample was collected and analyzed for the following biochemical parameters: NT-proBNP and intact PTH were estimated by sandwich immunoassay, and total 25-hydroxyvitamin D was measured by competitive immunoassay on Cobas e411 (Roche Diagnostics Mannheim Germany).

Serum albumin and creatinine were measured by bromocresol green and Jaffe's rate blank colorimetric assay respectively; serum alkaline phosphatase was estimated by International Federation of Clinical Chemistry method; serum calcium, phosphorus, and magnesium were measured by colorimetric assays on Cobas C501 (Roche Diagnostics Mannheim Germany).

Echocardiography was performed by the same cardiologist for all these patients. All samples were analyzed on the same day of collection.

\section{Statistical Analysis}

As the data are not normally distributed, it is presented as medians and interquartile range for continuous variables. Mann-Whitney $U$ test is used to compare cases with controls. Demographic, clinical, and laboratory characteristics were compared across quartiles of PTH by Kruskal-Wallis test for continuous variables. Logistic regression was used to correlate the association between serum PTH and congestive heart failure (CHF).

All reported $p$-values are two-tailed, with $p<0.05$ indicating statistical significance. Analysis was performed using MedCalc version 16.8 software.

\section{RESULTS}

The study group consisted of 80 males and 40 females, with mean age group of 56 years. These cases were compared with 60 age- and sex-matched controls.

The mean NT-proBNP and PTH are $3752 \mathrm{pg} / \mathrm{mL}$ and $81.5 \mathrm{pg} / \mathrm{mL}$ respectively, which is higher in cases as compared with controls and is statistically significant.

The median LVEF is 30\%, which is lower than controls and is statistically significant. The median vitamin $\mathrm{D}$ concentration in cases is $18 \mathrm{ng} / \mathrm{mL}$ and this is lower than the control group, but is not statistically significant (Graph 1).

The median alkaline phosphatase, creatinine, and phosphorus concentrations are $97 \mathrm{U} / \mathrm{L}, 1.2 \mathrm{mg} / \mathrm{dL}$, and $4.2 \mathrm{mg} / \mathrm{dL}$ respectively, which are significantly higher in cases compared with controls.

The median serum albumin, calcium, and magnesium are $3.3 \mathrm{~g} / \mathrm{dL}, 9 \mathrm{mg} / \mathrm{dL}$, and $0.6 \mathrm{mmol} / \mathrm{L}$ respectively, and are significantly lower in cases compared with controls. A total of $104(86.6 \%)$ cases had vitamin D insufficiency and $100(83.3 \%)$ subjects have PTH concentration above the normal range (Table 1 ).

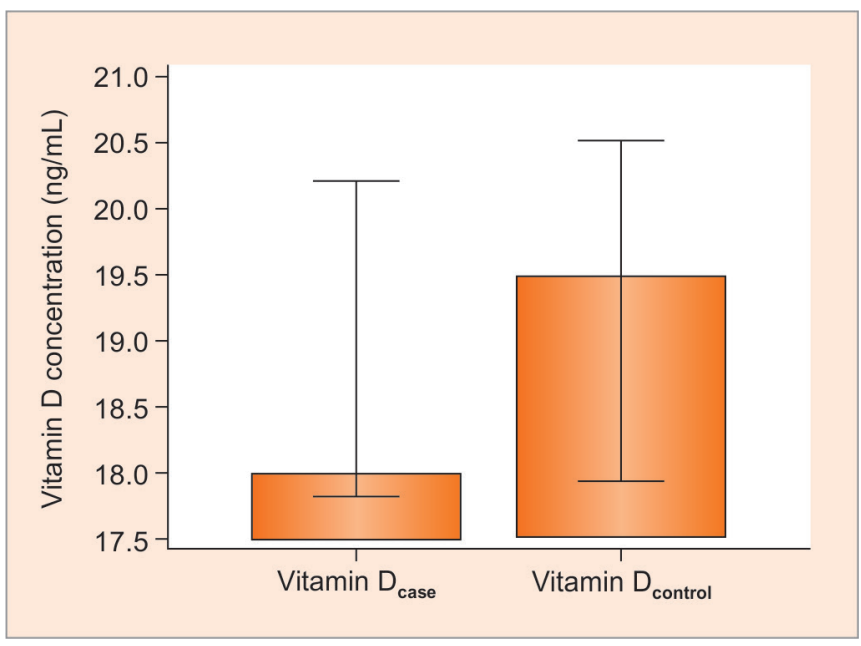

Graph 1: Concentration of Vitamin D in cases and controls

Table 1: Baseline characteristics of variables in study groups

\begin{tabular}{lllr}
\hline Variable & Cases & Controls & $p$-value \\
\hline Number of subjects & $\mathrm{n}=120$ & $\mathrm{n}=60$ & $\mathrm{NS}$ \\
Age $($ years) & $55(50-62)$ & $53(50-58)$ & $<0.0001$ \\
Albumin $(\mathrm{g} / \mathrm{dL})$ & $3.3(3-3.5)$ & $3.9(3.5-4.4)$ & $<0.0001$ \\
Alkaline phosphatase $(\mathrm{U} / \mathrm{L})$ & $97(81-111)$ & $73(66-84)$ & $<0.0001$ \\
Calcium $(\mathrm{mg} / \mathrm{dL})$ & $9(8.7-9.2)$ & $9.4(9-9.6)$ & $<0.0001$ \\
Creatinine $(\mathrm{mg} / \mathrm{dL})$ & $1.2(1.1-1.5)$ & $0.9(0.8-1.1)$ & $<0.0001$ \\
LVEF $(\%)$ & $30(26-40)$ & $60(58-63)$ & $<0.0001$ \\
Magnesium $(\mathrm{mmol} / \mathrm{L})$ & $0.6(0.6-0.8)$ & $1.1(1-1.2)$ & $<0.0001$ \\
NT-proBNP $(\mathrm{pg} / \mathrm{mL})$ & $3752(1078-10857)$ & $54(34-55.6)$ & $<0.0001$ \\
Phosphorus $(\mathrm{mg} / \mathrm{dL})$ & $4.2(3.6-4.8)$ & $2.9(2.5-3.3)$ & $<0.0001$ \\
PTH $(\mathrm{pg} / \mathrm{mL})$ & $81.5(67-117)$ & $34.5(31.4-42)$ & $\mathrm{NS}$ \\
Vitamin D $(\mathrm{ng} / \mathrm{mL})$ & $18(15-27)$ & $19.5(15-22)$ & \\
\hline Val & &
\end{tabular}

Values expressed are median (interquartile range); NS: Not significant 
Study of PTH as an Independent Risk Marker of HF

Table 2: Baseline demographics according to quartiles of PTH

\begin{tabular}{|c|c|c|c|c|c|}
\hline Variable & $\begin{array}{l}\text { Lower quartile, } \\
\text { median }=64.14 \mathrm{pg} / \mathrm{mL} \text {, } \\
\text { range } 34.4-67\end{array}$ & $\begin{array}{l}\text { Second quartile, } \\
\text { median }=75.31 \mathrm{pg} / \mathrm{mL} \text {, } \\
\text { range } 68-78\end{array}$ & $\begin{array}{l}\text { Third quartile, } \\
m=114 \mathrm{pg} / \mathrm{mL}, \\
\text { range } 85-117\end{array}$ & $\begin{array}{l}\text { Highest quartile, } \\
\text { median }=263 \mathrm{pg} / \mathrm{mL} \text {, } \\
\text { range } 118-285\end{array}$ & $p$-value \\
\hline Number of patients & 30 & 30 & 30 & 30 & \\
\hline Age; years (interquartile range) & $55(54-58)$ & $65(49-70)$ & $58(50-60)$ & $52(48-62)$ & 0.02 \\
\hline Male gender $(\%)$ & 63.40 & 53 & 56.70 & 76.70 & \\
\hline \multicolumn{6}{|l|}{ NYHA class (\%) } \\
\hline II & $11(35.48)$ & $16(51.61)$ & $4(12.9)$ & 0 & \\
\hline III & $15(37.5)$ & $9(22.5)$ & $7(17.5)$ & $9(22.5)$ & \\
\hline IV & $4(8.16)$ & $4(8.16)$ & $20(40.8)$ & $21(42.85)$ & \\
\hline LVEF (\%) & $35(30-45)$ & $45(26-49)$ & $25(25-34)$ & $30(30-34)$ & $<0.0001$ \\
\hline \multicolumn{6}{|c|}{ Laboratory values (interquartile range) } \\
\hline Albumin (g/dL) & $3.4(3.3-3.5)$ & $3(2.8-3.2)$ & $3.7(3.3-3.9)$ & $3.2(3-3.5)$ & $<0.0001$ \\
\hline Alkaline phosphatase (U/L) & $90(81-92)$ & $113(100-149)$ & $97.5(81-98)$ & $95(78-111)$ & $<0.0001$ \\
\hline Calcium (mg/dL) & $9.5(9.3-9.7)$ & $9.1(8.9-9.5)$ & $9.4(9.3-9.8)$ & $9.2(9-9.5)$ & $<0.0001$ \\
\hline Creatinine (mg/dL) & $1.2(1.1-1.4)$ & $1.4(1-1.7)$ & $1.4(1.1-1.6)$ & $1.2(1.1-1.3)$ & NS \\
\hline Hemoglobin (g/dL) & $12.8(11-14)$ & $11.3(11-11.5)$ & $13(12.8-13)$ & $11(11-16)$ & $<0.0006$ \\
\hline Magnesium (mmol/L) & $0.6(0.6-0.7)$ & $0.6(0.5-0.6)$ & $0.7(0.6-0.8)$ & $0.8(0.5-0.9)$ & $<0.0004$ \\
\hline NT-proBNP (pg/mL) & 1072 (940-2620) & $6724(4622-27270)$ & $3652(3553-5638)$ & 17190 (2937-18777) & $<0.0001$ \\
\hline Phosphorus (mg/dL) & $3.6(3.1-4.3)$ & $4(3.9-6.4)$ & $4.4(3.6-5)$ & $4.2(3.7-4.6)$ & $<0.002$ \\
\hline Vitamin D (ng/mL) & $16(14-17.2)$ & $22.2(15-38.6)$ & $22.4(18-27)$ & $18(14-19)$ & $<0.001$ \\
\hline
\end{tabular}

Values expressed are median (interquartile range)

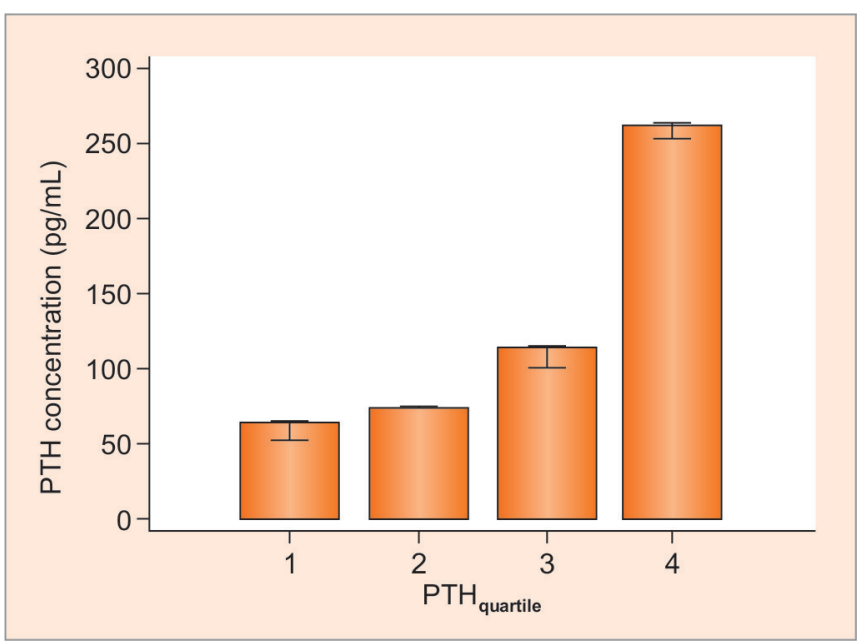

Graph 2: Concentration of PTH in different quartiles

Demographic, clinical, and laboratory data were compared across quartiles of PTH. Significant difference is found in age, $\mathrm{LVEF} \%$, albumin, alkaline phosphatase, calcium, phosphorus, magnesium, NT-proBNP, and vitamin D across the quartiles of PTH (Table 2).

The mean concentration of PTH across different quartiles is depicted in Graph 2.

Highest quartile of PTH has more of NYHA class IV HF cases compared with other quartiles, indicating high PTH is associated with severe disease, which accounts for $42.85 \%(n=21)$ of total class IV patients in the study.

The concentration of PTH showed significant positive correlation with NT-proBNP, $\mathrm{r}=0.409$ ( $\mathrm{p}<0.001 ; 95 \% \mathrm{CI}$ $0.248-0.548$ ), and negative correlation with vitamin $\mathrm{D}$,
Table 3: Correlation for PTH

\begin{tabular}{llcl}
\hline $\begin{array}{l}\text { Dependent } \\
\text { variable }\end{array}$ & Variable & $\begin{array}{l}\text { Correlation } \\
\text { coefficient } r\end{array}$ & $p$-value \\
\hline PTH & Age & -0.06 & NS \\
& NT-proBNP & 0.41 & $<0.0001$ \\
& Vitamin D & -0.26 & $<0.0001$ \\
& EF\% & -0.343 & 0.0001 \\
& Alkaline phosphatase & -0.078 & NS \\
& Calcium & -0.016 & NS \\
& Hemoglobin & -0.035 & NS \\
\hline
\end{tabular}

NS: Not significant

$\mathrm{r}=-0.26(\mathrm{p}<0.001,95 \% \mathrm{CI}-0.419$ to -0.084$)$ and LVEF, $r=-0.343(p<0.0001,95 \%$ CI -0.492 to 0.17$)$ (Table 3).

Logistic regression analysis demonstrated that high concentration of PTH (odds ratio of 1.1113; 95\% CI 1.07-1.14; $\mathrm{p}<0.0001$ ) and low levels of vitamin D (odds ratio of 1.053 ; $95 \%$ CI $1.0079-1.1009$ ) are significantly associated with $\mathrm{HF}$.

\section{DISCUSSION}

Heart failure is a syndrome with considerable morbidity and mortality. Hyperparathyroid disorders are associated with osteoporosis and altered mineral metabolism. Increased PTH concentration is responsible for overloading of calcium in cardiomyocytes through a receptormediated influx of calcium, which eventually results in necrosis of the cell and causes worsening of the cardiac function. ${ }^{8}$

Parathyroid hormone has been shown to be an independent predictor of all-cause and cardiovascular 
mortality in patients with HF.7 In this study, we found that high PTH is significantly associated with HF and can be considered as independent risk factor for CHF. These findings are consistent with a community-based study by Hagström et al, ${ }^{9}$ who showed that PTH predicts HF hospitalization and is an independent risk factor for HF. Several factors are responsible for increase in PTH concentration in HF. Firstly, there is an increased calcium loss and impaired cation homeostasis, as a consequence of hormonal changes (hyperadrenergic state and secondary hyperaldosteronism). ${ }^{10,11}$

Calcium loss is also triggered by diuretics used to treat $\mathrm{HF}_{,}^{12}$ although conflicting results have also been reported. ${ }^{11}$ In our study, we observed a normal serum calcium concentration.

Secondly, a low concentration of vitamin D can also lead to increased concentration of $\mathrm{PTH} .{ }^{13-15}$ In our study, $98(81.67 \%)$ patients are vitamin D insufficient. This is consistent with Schierbeck et al, ${ }^{7}$ who have reported a vitamin D insufficiency of $43 \%$ in their study subjects. Vitamin D deficiency is also associated with increased arterial wall stiffness in young population and this is independent of PTH concentration. The higher number of insufficiency in our study group is due to the deficiency of vitamin D seen in our population. High PTH has deleterious effect on heart by several mechanisms. High PTH causes endothelial dysfunction and atherosclerosis causing cardiac ischemia and consequently HF.

Parathyroid hormone has direct detrimental effect on myocardium as PTH receptors are also found on myocardium and high PTH may induce myocyte hypertrophy, fibrosis, left ventricular hypertrophy, and nonischemic HF. ${ }^{9}$

High concentration of PTH is associated with severity of CHF. There are several studies that have shown that high PTH concentration is associated with severity of disease indicated by NYHA functional classification and LVEF\% ${ }^{13,16}$ In our study, we have also found that NYHA class IV patients (43\%) are more in highest quartiles of PTH. Further, the EF\% shows a significant negative correlation with $\mathrm{PTH}$ concentration, indicating that more severe form of disease is associated with high PTH. Circulating PTH levels also showed significant correlation with NT-proBNP levels. NT-proBNP is produced primarily within heart and is released into circulation in response to increased wall tension and is a sensitive marker of HF. Several studies have shown that high concentration of PTH is a predictor of hospitalization. ${ }^{8,9}$

The predictive value of PTH in cardiovascular and all-cause mortality in HF has been documented to be independent of known risk factors, such as estimated glomerular filtration rate, LVEF, NT-proBNP, and age. ${ }^{17}$
The cardiac impact of PTH is also related to calcium overloading in myocardial cells.

Hyperparathyroidism has also been documented to trigger oxidative stress. ${ }^{4}$ When PTH levels are increased, a high $\mathrm{H}_{2} \mathrm{O}_{2}$ production is observed in peripheral mononuclear cells. ${ }^{4,10}$ The increased intracellular calcium induced by PTH might impair the mitochondrial function and ATP production, inducing reactive oxygen species and leading to oxidative stress as well as inflammation and in the end to cardiomyocyte necrosis. ${ }^{10,11}$

Parathyroid hormone is found to stimulate the adrenal aldosterone synthesis. ${ }^{17-19}$ A relative aldosterone excess can cause sodium retention and oxidative stress. ${ }^{20,21}$

The significance of demonstration of association of PTH and HF is that it can be an adjustable factor in the setting of low vitamin D concentration. In our study group, $83 \%$ of subjects have PTH above the normal range, while none of them have primary hyperparathyroidism. The secondary hyperparathyroidism found in these cases can be due to vitamin D insufficiency, which can be corrected with vitamin $\mathrm{D}$ supplementations.

Although large numbers of studies are available suggesting a causal role of high PTH in HF and our study also shows the same, there is no available evidence to suggest that decreasing PTH concentration will reduce the risk of HF. More interventional studies are needed to evaluate the potential role of PTH lowering to prevent HF.

\section{CONCLUSION}

Higher concentration of PTH is associated with severe form of HF. Parathyroid hormone estimation can help in risk stratification of CHF patients. Vitamin D insufficiency is seen in the study group; supplementation of vitamin D may be beneficial to the patients with HF.

\section{LIMITATION}

Due to relatively small sample size of the study group, the results cannot be generalized, and milder cases that do not need hospitalization are not included in the study.

\section{REFERENCES}

1. Mosterd A, Hoes AW. Clinical epidemiology of heart failure. Heart 2007 Sep;93(9):1137-1146.

2. Braunwald E. Biomarkers in heart failure. N Engl J Med 2008 May;358:2148-2159.

3. Bansal N, Zelnick L, Robinson-Cohen C, Hoofnagle AN, Ix JH, Lima JA, Shoben AB, Peralta CA, Siscovick DS, Kestenbaum B, et al. Serum parathyroid hormone and 25-hydroxy vitamin $\mathrm{D}$ concentrations and risk of incident heart failure: the multi-ethnic study of atherosclerosis. J Am Heart Assoc 2014 Dec;3(6):e001278.

4. Gruson D, Buglioni A, Burnett JC. PTH: potential role in management of heart failure. Clin Chim Acta 2014 Jun;433:290-296. 
5. Pepe J, Romagnoli E, Nofroni I, Pacitti MT, De Ceronimo S, Letizia C, Tonnarini C, Scarpiello A, D’Erasmo E, Minisola S. Vitamin D status as the major factor determining the circulating levels of parathyroid hormone: a study in normal subjects. Osteoporos Int 2005 Jul;16(7):805-812.

6. Shimoyama M, Ogino K, Furuse $Y$, Uchida K, Kinugasa $Y$, Tomikura Y, Igawa O, Hisatome I, Bilezikian JP, Shigemasa C. Signaling pathway and chronotropic action of parathyroid hormone in isolated perfused rat heart. J Cardiovasc Pharmacol 2001 Oct;38(4):491-499.

7. Schierbeck LL, Jensen TS, Bang U, Jensen G, Køber L, Jensen JE. Parathyroid hormone and vitamin D-markers for cardiovascular and all cause mortality in heart failure. Eur J Heart Fail 2011 Jun;13(6):626-632.

8. Fujita T, Palmieri GM. Calcium paradox disease: calcium deficiency prompting secondary hyperparathyroidism and cellular calcium overload. J Bone Miner Metab 2000 Feb;18(3):109-125.

9. Hagström E, Ingelsson E, Sundström J, Hellman P, Larsson TE, Berglund L, Melhus H, Held C, Michaëlsson K, Lind L, et al. Plasma parathyroid hormone and risk of congestive heart failure in the community. Eur J Heart Fail 2010 Nov;12(11):1186-1192.

10. Tomaschitz A, Ritz E, Pieske B, Rus-Machan J, Kienreich K, Verheyen N, Gaksch M, Grübler M, Fahrleitner-Pammer A, Mrak $\mathrm{P}$, et al. Aldosterone and parathyroid hormone interactions as mediators of metabolic and cardiovascular disease. Metabolism 2014 Jan;63(1):20-31.

11. TomaschitzA, RitzE,PieskeB,Fahrleitner-PammerA,KienreichK, Horina JH, Drechsler C, März W, Ofner M, Pieber TR, et al. Aldosterone and parathyroid hormone: a precarious couple for cardiovascular disease. Cardiovasc Res 2012 Apr;94(1):10-19.

12. Law PH, Sun Y, Bhattacharya SK, Chholar VS, Weber KT. Diuretics and bone loss in rats with aldosteronism. J Am Coll Cardiol 2005 Jul;46(1):142-146.

13. Gruson D, Lepoutre T, Ahn SA, Ketekslegers JM, Rousseau MF. Increased circulating concentrations of bioactive PTH1-84 in patients with heart failure. J Endocrinol Invest 2012 Dec;35(11):987-991.

14. Tomachitz A, Pilz S, Ritz E, Meinitzer A, Boehm BO, März W. Plasma aldosterone levels are associated with increased cardiovascular mortality: the Ludwigshafen Risk and Cardiovascular Health (LURIC) study. Eur Heart J 2010 May;31(10):1237-1247.

15. Bozic B, Loncar G, Prodanovic N, Lepic T, Radojicic Z, Cvorovic V, Dimkovic S, Popovic V. Parathyroid hormone response to vitamin $\mathrm{D}$ insufficiency in elderly males with chronic heart failure. Physiol Res 2011 Jul;60(Suppl 1): S155-S163.

16. Altay H, Zorlu A, Binici S, Bilgi M, Yilmaz MB, Colkesen $Y$, Erol T, Muderrisoglu H. Relation of serum parathyroid hormone level to severity of heart failure. Am J Cardiol 2012 Jan;109(2):252-256.

17. Chhokar VS, Sun Y, Bhattacharya SK, Ahokas RA, Myers LK, Xing Z, Smith RA, Gerling IC, Weber KT. Hyperparathyroidism and the calcium paradox of aldosteronism. Circulation 2005 Feb;111(7):871-878.

18. Rutledge MR, Farah V, Adebpye AA, Seawell MR, Bhattacharya SK, Weber KT. Parathyroid hormone a crucial mediator of pathologic cardiac remodelling in aldosteronism. Cardiovasc Drugs Ther 2013 Apr;27(2):161-170.

19. Vidal A, Sun Y, Bhattacharya SK, Ahokas RA, Gerling IC, Weber KT. Calcium paradox of aldosteronism and the role of the parathyroid glands. Am J Physiol Heart Circ Physiol 2006 Jan;290(1):H286-H294.

20. Sowesr JR, Whaley-Connell A, Epstein M. Narrative review: the emerging clinical implications of the role of aldosterone in the metabolic syndrome and resistant hypertension. Ann Intern Med 2009 Jun;150(11):776-783.

21. Beygui F, Collet JP, Benoleil JJ, Vignolles N, Dumaine R, Barthélémy O, Montalescot G. High plasma aldosterone levels on admission are associated with death in patients presenting with acute ST-elevation myocardial infarction. Circulation 2006 Dec;114(24):2604-2610. 